\title{
Incidence and distribution of Lemon butterfly (Papilio demoleus L.) on five alternate Citrus hosts at Sahati region, Sindh-Pakistan
}

\author{
Wali Muhammad Mangrio', Hakim Ali Sahito ${ }^{1,2^{*}}$, Bhugro Mal $^{3}$, \\ Tasneem Kousar ${ }^{1}$, Zafar Hussain Shah ${ }^{1}$ and Faheem Ahmed Jatoi ${ }^{1}$ \\ 1. Department of Zoology, Faculty of Natural Sciences, Shah Abdul Latif University, Khairpur Mir's, 66111 \\ Sindh-Pakistan \\ 2. Date Palm Research Institute (DPRI), Shah Abdul Latif University, Khairpur Mir's, 66111 Sindh-Pakistan \\ 3. Southern Zone Agricultural Research Centre, PARC, Karachi, 75270 Sindh-Pakistan \\ *Corresponding author's email: hakim.sahito@salu.edu.pk
}

Citation

Wali Muhammad Mangrio, Hakim Ali Sahito, Bhugro Mal, Tasneem Kousar, Zafar Hussain Shah and Faheem Ahmed Jatoi. Incidence and distribution of Lemon butterfly (Papilio demoleus L.) on five alternate Citrus hosts at Sahati region, Sindh-Pakistan. Pure and Applied Biology. Vol. 9, Issue 4, pp2637-2647.

http://dx.doi.org/10.19045/bspab.2020.90280

Received: 19/03/2020 Revised: 30/05/2020

Accepted: 02/08/2020

Online First: 17/08/2020

\section{Abstract}

In Sindh province of Pakistan district, Naushahro Feroze is considered as the main hub for citrus fruit productivity. At field conditions research studies were carried out from five different citrus farmlands of the given Sahati region. All life cycle stages of Papilio demoleus were observed from the $C$. paradisi, $C$. sinensis, $C$. limetta, $C$. reticulata and $C$. aurantium. During one-year study plan data were taken fortnightly interval basis from the host plants. It was frequently observed that temperature fluctuation relaying the effect on the population of the lemon butterfly during severe cold as well as in intense warmer conditions. The overall mean of the egg population was observed at (2.88 to 5.39), larvae (1.12 to 3.89) pupae (0.56 to 1.00$)$ adult $(\hat{\delta}:$; ) male and female $(0.45$ to 1.04$)$, respectively. During the October month population found high and in December month low on the leaves of five citrus verities i.e., Grape fruit maximum and minimum at (4.71 to 4.79), (1.27 to 1.39), Sweat orange (3.88 to 4.19), (1.12 to 1.38 ), Mousambi leaves (3.30 to 3.51), (0.91 to 1.03), Mandarian orange leaves (2.86 to 2.96$)$, (0.78 to 0.84 ) and Bitter orange leaves (2.33 to 2.46), (0.54 to 0.63 ) respectively. It was concluded that caterpillars' fourth and fifth stages are a voracious feeder of citrus orchard leaves. The main motto of the present work is to take initiative over the lemon butterfly and aware of citrus growers of this region. It is recommended and immediate call to enhance stable integrated pest management eco-friendly techniques to secure the citrus industry for the upcoming period.

Keywords: Caterpillars; Citrus orchards; Papilio demoleus; Sahati region; Voracious feeder Introduction

Citrus, the acidic world-leading fruit contains nutrients for the body healthier to man. Their fruits are not merely refreshing and delicious but richest sources of minerals, vitamins, and several other body essentials [1]. Tropical and subtropical areas of the world are sweat able for their magnificent growth and fruit production. These fruits are very commercial and had 
been cultivated on a large scale in the world for decades [2]. Citrus fruits are popularly denoted as Asian fruits but this economically valuable and juicy laden fruit crops grown also in many parts of the world where the climatic and soil condition are fruitful these fruits can grow and fruit production is gradually increasing [3]. Varieties of citrus fruits are severely harmed by insect pests from immaturity till to mature-hood of the plants.

Insects are the largest in number among all other living animals and have supremacy on the earth's planet on species basis [4]. Generally, agri-ecosystem is a favourable habitat for their survival activities and incrassation to their progeny [5]. Lepidopteron is a widely recognized and distributed order of insects throughout the world and generally regarded as a symbol of beauty, attractive, beautiful, magnificent, and amazing than other insects possessing captivated human mind's eye and vision [6]. They possess a high sense of power to recognize rapidly climatic change, solar radiation, temperature fluctuations, host plant better oviposition, and smooth larval development. All species of butterflies at their larval stages herbivores in their feeding behaviour and most of them have host-specific relationships to their host plants [7].

More than 250 reported species infest a variety of citrus fruits of which few species cause regular and severe damage but the lemon butterfly is worldwide pest insect to citrus orchards and cause constraints in a variety of citrus fruit production [8]. Citrus swallowtail butterfly found an association at their host plants. Citrus fruits are mostly attacked by the black fly, leaf miner, mites, fruit sucking moth and bark eating caterpillar [9] but $P$. demoleus, is economically considerable harmful insects badly hits to the maximum quantity of foliage of Rhamnaceae, Apiaceae, Rutaceae, Anacardiaceae, Fabaceae, and Sapindaceae family and their completely cause defoliation to young plants [10].

When an infestation occurs at wide range host plants retarded their growth and decrease yield quantity in the world but in the case of South-eastern Asia these pest insects found at the extensive range and wide dispersal area and fifth caterpillar badly hit to citrus orchards [11]. $P$. demoleus, at the larval stage, shows severity impacts on the growth stage of host plants at this vegetative stage host plants may completely defoliate [12]. The peak period for survival activity of lemon butterfly when the emergence of new foliage and lush green leaves occurs [13] and their caterpillar frequently found new growing citrus plantation especially when orchards at few feet in height [14]. There is many scientific documentary and reports of a severe infestation of lemon butterfly which ranges from Central America to the Caribbean but in case of Pakistan this pest insects occurring at wide ecological range due to fruitful climatic conditions which allow them tolerance ability and can move at a short distance and thousands of miles as well [15].

Adult lemon butterfly especially seeks host plants for oviposition and deposit their eggs under tender twigs and terminal parts of the shoots by using chemical cues. Those orchards which are best for larval growth adult female usually oviposit on it and during oviposition, female vibrate their wings [16]. Due to the extensive harmful hazardous of lemon butterfly at different citrus host plants, it is needed to formulate appropriate management to overcome form from economic losses of profitability and production of citrus crop. It is necessary for appropriate executive of any kind of insect pest to get appropriate knowledge about the feeding behaviour on different citrus fruits and biology of the insect but there is no adequate scientific documentary on the Papilio demoleus population on the different citrus host plants. 
Considering the different host plant preferences and to generate information about the bad impacts of the $P$. demoleus at different alternate citrus host plants present investigation was performed. This kind of research work was carried out first time from the district; Naushahro Feroze, Sindh-Pakistan to survey and find out the lemon butterfly pest population on different citrus plants and this work will be a supportive tool in the management of citrus insect pests.

The present work emphasizing the larval activities, effects on host plant association. In the present investigation, attention was taken to find out the LBF fluctuation in both seasons of the year and their developmental behaviours. Keeping these viewpoints in mind present research was conducted to find out the burden of $P$. demoleus at five varieties of citrus orchards of this region.

\section{Materials and methods}

\section{Study site}

An adult butterfly shows migratory behaviour due to phytophagous nature. They search food sources with the help of paired clavately/cubed antennae and receptors in their feet, due to this they move from one host plant to another. For the purpose to find out the incidence and distribution of LBF, the research study was carried out fortnightly basis to evaluate the alternate host plants of lemon butterfly at five different talukas namely; Meharabpur, Kandiaro, Naushahro Feroze, Bhiria and Moro of District; Naushahro Feroze during January to December 2019, to determine the larval infestation on the newly emerging leaves of Citrus paradisi, Citrus sinensis, Citrus limetta, Citrus reticulata, and Citrus aurantium.

\section{Selection of host citrus plants}

Through randomized selection, each variety of host citrus plants out of $(n=20)$ orchards only $(n=05)$ host plants were examined during a field survey at farmlands and these plants were also tagged for the next survey. Same way on fortnightly throughout the year data of the pest were taken from the given district.

Incidence of lemon butterfly population

During research work, all the stages of the pest insect, LBF from eggs, larval stages, pre-pupae, pupae, and adults were observed from the five varieties of citrus orchards. The pest population of the lemon butterfly was gathered and observed north, east, west, south and central canopy of the host orchards.

\section{Sampling procedure from infested leaves of host plants}

For the purpose to find out the intensity LBF pest population host plants i e., grapefruit were selected and observed from taluka; Mehrabpur, the distance from the plant to plant were measured $20 \mathrm{ft}$ and randomly selected five plants from different angles. Another host plant, sweat oranges were observed form taluka; Kandiaro and at farmland and distance from one plant to another measured $20 \mathrm{ft}$. Through randomly five plants were observed form alternate angels. The mousambi host plants were observed form taluka; Bhiria, found positive with a population of LBF, through random selection data were collected and $20 \mathrm{ft}$ distance between plants to plant was observed. From taluka, Naushahro Feroze, mandarin orange host plants were observed to check out the lemon butterfly larval burden from farmland and distance between the one orchard to another measured $18 \mathrm{ft}$. Bitter oranges host plants were examined form, taluka; Moro for the prevalence of $\mathrm{LBF}$ population and at farmland $15 \mathrm{ft}$ distance was measured from plant to plant.

\section{Statistical analysis}

All the data which were gathered from the different varieties of the host plants at different locations were statistically analysed and the analysis of variance (ANOVA) was conducted through random complete block design (RCBD) of different stages of LBF and for interpretation of results LSD means were 
separated by the application of student package 8.1 USA software.

\section{Results}

\section{The infestation of the lemon butterfly on} Grapefruit orchards

From the taluka, Meharabpur, the appearance of the LBF population was found on grapefruit leaves with significant differences. It was frequently observed when there is coldness and hottest months of the year pest population on the host plants decreases due to the intensity of temperature but when the temperature is moderate they rapidly increase their number. In the initial fifteen days of the December month, the minimum population including different stages were found at an overall mean (1.27) and maximum found during the early days of the October month at (4.79) with a least significant difference in each citrus verities $\mathrm{c}$ for $\mathrm{a}$ and $\mathrm{c}$ for $\mathrm{b}$ at grapefruit shows; $\quad(\mathrm{DF}=8 ; \quad \mathrm{F}=234.1$; $\mathrm{P}=0.00)$ and $(\mathrm{DF}=23 ; \mathrm{F}=41.20 ; \mathrm{P}=0.00)$ respectively, described (Table 1).

The infestation of the lemon butterfly on Sweat orange leaves

LBF population was observed another location, from the host sweat orange leaves at taluka, Kandiaro, and found with pest infestation. The minimum prevalence of pest was observed in the month of early December days at (1.12) average and maximum in October at (4.19) respectively. LSD of pairwise group shows at sweat orange leaves, $(\mathrm{DF}=8 ; \mathrm{F}=255.54$; $\mathrm{P}=0.00)$ and $(\mathrm{DF}=23 ; \mathrm{F}=40.68 ; \mathrm{P}=0.00)$ respectively, shown in (Table 2 ).

The infestation of the lemon butterfly on Mousambi leaves

Mousambi leaves were observed for the presence of the LBF population from the form land at taluka, Bhiria. All stages of pest population were found and minimum infestation observed in December at an overall mean of (0.91) and maximum during October at (3.51). Treatment shows significant difference among pairwise treatments on mousambi leaves, $(\mathrm{DF}=8$; $\mathrm{F}=349.06 ; \mathrm{P}=0.00)$ and $(\mathrm{DF}=23 ; \mathrm{F}=84.74$;
$\mathrm{P}=0.00)$ respectively, description given in (Table 3).

The infestation of the lemon butterfly on Mandarian orange leaves

For the prevalence of LBF, mandarin orange leaves were observed from another farmland, at taluka, Naushahro Feroze, and reported all different stages of the LBF pest population. Minimum prevalence was found in December and the maximum in October at (0.78 to 2.96). LSD shows least significant difference of infestation at mandarin orange leaves, $\quad(\mathrm{DF}=8$; $\mathrm{F}=268.37 ; \mathrm{P}=0.00)$ and $(\mathrm{DF}=23 ; \mathrm{F}=40.76$; $\mathrm{P}=0.00$ ) respectively, given in (Table 4).

The infestation of the lemon butterfly on Bitter orange leaves

Form the farmland at taluka, Moro, host plants Bitter orange leaves were examined for LBF prevalence and found all stages of pest population on the leaves of the host plants. Pest population observed minimum on the host plants during the last month of the year as compared to other months, while as the maximum in October compared to other months minimum and maximum at ( 0.54 to 2.46$)$ overall mean. Also found significant difference at $(\mathrm{DF}=8 ; \mathrm{F}=189.82 ; \mathrm{P}=0.00)$ and $(\mathrm{DF}=23$; $\mathrm{F}=37.89 ; \mathrm{P}=0.00)$ respectively, described in (Table 5).

The overall mean population of the Lemon butterfly on different Citrus host plants

In the present research study, it was observed the positive incidence of LBF pest population on all five selected verities of citrus fruits throughout the year during 2019. But the pest population was found with fluctuations. Maximum and minimum pest populations were reported from the host plant leaves. In one year study overall mean of eggs population were found minimum and maximum at (2.88), (3.70), (4.43), (4.83), (5.39) larval population at (1.12 to 2.06$),(1.24$ to 2.62$)$, (1.56 to $2.81)$, (1.65 to 3.28$)$ and (2.22 to 3.89$)$ respectively. While; pupae at (0.56), (0.67), (0.78), (0.89), (1.00) and adult male/female population at (0.45 to 0.65$)$, 
(0.54 to 0.75$),(0.58$ to 0.82$),(0.66$ to $0.89)$ and $(0.78$ to 1.04$)$ respectively. $1^{\text {st }}$ stage larvae show a maximum in their number as compared to other stages of larvae but the population of eggs was greater than the population of all larvae stages in given verities. When data were analysed that shows a significant difference among the egg to adult stages on five citrus varieties. All pairwise compressions show the least significant difference among whole treatments and in alphabetic letters, their justification has been given in (Fig. 1).

Table 1. Overall mean and SD of different stages of LBF on host plants, Citrus paradisi leaves under field conditions

\begin{tabular}{|c|c|c|c|c|c|c|c|c|c|c|}
\hline Months & Eggs & $\begin{array}{c}1^{\text {st }} \\
\text { instar }\end{array}$ & $\begin{array}{c}2^{\text {nd }} \\
\text { instar }\end{array}$ & $\begin{array}{c}3^{\text {rd }} \\
\text { instar }\end{array}$ & $\begin{array}{c}4^{\text {th }} \\
\text { instar }\end{array}$ & $\begin{array}{c}5^{\text {th }} \\
\text { instar }\end{array}$ & Pupae & $\begin{array}{c}\text { Adult } \\
\text { male }\end{array}$ & $\begin{array}{l}\text { Adult } \\
\text { female }\end{array}$ & Mean \pm SD \\
\hline Jan.01 & 3.40 & 2.30 & 1.70 & 1.60 & 1.20 & 1.10 & 0.50 & 0.30 & 0.50 & $1.40 \pm 0.06^{\mathrm{lm}}$ \\
\hline Jan.02 & 3.60 & 2.70 & 1.90 & 1.80 & 1.40 & 1.30 & 0.60 & 0.30 & 0.70 & $1.59 \pm 0.03^{\mathrm{klm}}$ \\
\hline Feb.01 & 4.20 & 3.50 & 2.60 & 2.20 & 1.60 & 1.50 & 0.70 & 0.40 & 0.80 & $1.94 \pm 0.04 \mathrm{f}^{\text {ghijk }}$ \\
\hline Feb.02 & 4.40 & 3.20 & 2.70 & 2.50 & 2.00 & 1.90 & 0.80 & 0.40 & 0.60 & $2.06 \pm 0.08^{\text {fghij }}$ \\
\hline Mar.01 & 6.10 & 4.40 & 3.60 & 3.40 & 2.80 & 2.40 & 1.10 & 0.60 & 1.00 & $2.82 \pm 0.06^{\text {cde }}$ \\
\hline Mar.02 & 6.30 & 3.50 & 3.40 & 3.50 & 2.90 & 2.50 & 1.10 & 0.60 & 0.80 & $2.73 \pm 0.05^{\mathrm{de}}$ \\
\hline Apr.01 & 6.20 & 3.80 & 3.90 & 3.70 & 3.10 & 2.60 & 1.20 & 0.70 & 1.10 & $2.92 \pm 0.05^{\mathrm{cd}}$ \\
\hline Apr.02 & 6.50 & 4.50 & 3.70 & 3.60 & 3.20 & 2.70 & 1.10 & 0.80 & 1.00 & $3.01 \pm 0.06^{\mathrm{cd}}$ \\
\hline May.01 & 5.30 & 4.00 & 3.10 & 2.80 & 2.20 & 1.80 & 0.90 & 0.60 & 0.80 & $2.39 \pm 0.06^{\mathrm{efg}}$ \\
\hline May.02 & 5.20 & 3.60 & 3.20 & 3.00 & 2.40 & 2.30 & 0.80 & 0.60 & 0.80 & $2.43 \pm 0.06^{\mathrm{ef}}$ \\
\hline June.01 & 3.60 & 2.50 & 1.90 & 1.70 & 1.30 & 1.20 & 0.60 & 0.40 & 0.60 & $1.53 \pm 0.04^{\mathrm{klm}}$ \\
\hline June.02 & 3.80 & 3.00 & 2.10 & 2.00 & 1.60 & 1.50 & 0.80 & 0.30 & 0.50 & $1.73 \pm 0.04^{\mathrm{ijkl}}$ \\
\hline July.01 & 3.80 & 2.70 & 2.10 & 1.90 & 1.40 & 1.30 & 0.60 & 0.40 & 0.60 & $1.64 \pm 0.03^{\mathrm{jklm}}$ \\
\hline July.02 & 4.00 & 3.20 & 2.30 & 2.20 & 1.80 & 1.70 & 0.80 & 0.30 & 0.50 & $1.87 \pm 0.04^{\text {hijk }}$ \\
\hline Aug.01 & 6.70 & 4.00 & 4.30 & 3.90 & 3.60 & 3.00 & 1.30 & 1.80 & 1.20 & $3.20 \pm 0.10^{\mathrm{c}}$ \\
\hline Aug.02 & 6.30 & 4.60 & 4.00 & 3.80 & 3.50 & 3.00 & 1.20 & 1.90 & 1.10 & $3.16 \pm 0.06^{\mathrm{cd}}$ \\
\hline Sep.01 & 8.20 & 5.80 & 5.20 & 4.60 & 4.10 & 3.70 & 1.60 & 1.70 & 1.90 & $4.09 \pm 0.11^{\mathrm{b}}$ \\
\hline Sep.02 & 7.80 & 6.40 & 4.80 & 4.30 & 4.10 & 3.60 & 1.50 & 2.00 & 2.20 & $4.08 \pm 0.10^{\mathrm{b}}$ \\
\hline Oct.01 & 9.60 & 6.70 & 5.80 & 5.00 & 4.60 & 4.30 & 2.10 & 2.30 & 2.70 & $4.79 \pm 107^{\mathrm{a}}$ \\
\hline Oct.02 & 8.70 & 7.20 & 5.50 & 4.90 & 4.60 & 4.10 & 2.00 & 2.50 & 2.90 & $4.71 \pm 0.07^{\mathrm{a}}$ \\
\hline Nov.01 & 4.80 & 3.40 & 2.80 & 2.40 & 1.80 & 1.60 & 0.80 & 0.60 & 0.80 & $2.11 \pm 0.06^{\text {fghi }}$ \\
\hline Nov.02 & 4.60 & 3.70 & 2.90 & 2.80 & 2.20 & 2.10 & 0.90 & 0.50 & 0.70 & $2.27 \pm 0.05^{\mathrm{fgh}}$ \\
\hline Dec.01 & 3.20 & 2.10 & 1.50 & 1.40 & 1.10 & 0.90 & 0.40 & 0.30 & 0.50 & $1.27 \pm 0.05^{\mathrm{m}}$ \\
\hline Dec.02 & 3.00 & 2.50 & 1.70 & 1.50 & 1.20 & 1.10 & 0.50 & 0.40 & 0.60 & $1.39 \pm 0.06^{1 \mathrm{~m}}$ \\
\hline
\end{tabular}

Table 2. Overall mean and SD of different stages of LBF on host plants, Citrus sinensis leaves under field conditions

\begin{tabular}{|c|c|c|c|c|c|c|c|c|c|c|}
\hline Months & Eggs & $\begin{array}{c}\mathbf{1}^{\text {st }} \\
\text { instar }\end{array}$ & $\begin{array}{c}\mathbf{2}^{\text {nd }} \\
\text { instar }\end{array}$ & $\begin{array}{c}\mathbf{3}^{\text {rd }} \\
\text { instar }\end{array}$ & $\begin{array}{c}\mathbf{4}^{\text {th }} \\
\text { instar }\end{array}$ & $\begin{array}{c}\mathbf{5}^{\text {th }} \\
\text { instar }\end{array}$ & Pupae & $\begin{array}{c}\text { Adult } \\
\text { male }\end{array}$ & $\begin{array}{c}\text { Adult } \\
\text { female }\end{array}$ & Mean \pm SD \\
\hline Jan.01 & 3.20 & 3.20 & 1.50 & 1.40 & 1.00 & 1.10 & 0.40 & 0.20 & 0.40 & $1.38 \pm 0.05^{\text {jk }}$ \\
\hline Jan.02 & 3.40 & 2.50 & 1.60 & 1.50 & 1.30 & 1.00 & 0.40 & 0.30 & 0.50 & $1.39 \pm 0.04^{\text {jk }}$ \\
\hline Feb.01 & 4.10 & 2.60 & 2.10 & 1.80 & 1.90 & 1.20 & 0.60 & 0.40 & 0.60 & $1.70 \pm 0.07^{\text {ghij }}$ \\
\hline Feb.02 & 3.80 & 2.50 & 1.70 & 1.80 & 1.60 & 1.00 & 0.70 & 0.30 & 0.50 & $1.54 \pm 0.05^{\text {hij }}$ \\
\hline Mar.01 & 5.00 & 3.60 & 2.80 & 2.60 & 2.50 & 1.80 & 1.00 & 0.60 & 0.80 & $2.30 \pm 0.06^{\text {de }}$ \\
\hline Mar.02 & 5.60 & 3.30 & 2.50 & 2.40 & 2.10 & 1.70 & 0.90 & 0.60 & 0.80 & $2.21 \pm 0.06^{\text {def }}$ \\
\hline Apr.01 & 5.90 & 3.80 & 3.00 & 2.80 & 2.80 & 2.10 & 1.10 & 0.70 & 1.10 & $2.59 \pm 0.04^{\text {cd }}$ \\
\hline Apr.02 & 5.50 & 3.60 & 2.80 & 2.70 & 2.40 & 2.00 & 1.00 & 0.70 & 0.90 & $2.40 \pm 0.05^{\text {cd }}$ \\
\hline May.01 & 4.40 & 3.00 & 2.40 & 2.20 & 2.30 & 1.50 & 0.80 & 0.50 & 0.70 & $1.98 \pm 0.04^{\text {efg }}$ \\
\hline May.02 & 4.60 & 2.90 & 2.20 & 2.10 & 1.90 & 1.40 & 0.70 & 0.50 & 0.70 & $1.89 \pm 0.03^{\text {fgh }}$ \\
\hline June.01 & 3.40 & 2.40 & 1.70 & 1.50 & 1.50 & 1.00 & 0.50 & 0.30 & 0.50 & $1.42 \pm 0.06^{\text {ijk }}$ \\
\hline
\end{tabular}


Mangrio et al.

\begin{tabular}{|c|c|c|c|c|c|c|c|c|c|c|}
\hline June.02 & 3.50 & 2.20 & 1.50 & 1.40 & 1.30 & 0.80 & 0.60 & 0.30 & 0.50 & $1.34 \pm 0.06^{\mathrm{jk}}$ \\
\hline July.01 & 3.50 & 2.50 & 1.80 & 1.60 & 1.70 & 1.10 & 0.50 & 0.30 & 0.50 & $1.50 \pm 0.07^{\mathrm{jjk}}$ \\
\hline July.02 & 3.60 & 2.30 & 1.60 & 1.50 & 1.40 & 0.90 & 0.70 & 0.30 & 0.50 & $1.42 \pm 0.05^{\mathrm{jjk}}$ \\
\hline Aug.01 & 5.70 & 4.10 & 3.50 & 3.10 & 3.00 & 2.40 & 1.30 & 0.80 & 1.00 & $2.77 \pm 0.09^{\mathrm{c}}$ \\
\hline Aug.02 & 6.40 & 3.70 & 3.30 & 3.00 & 2.70 & 2.40 & 1.20 & 0.80 & 1.00 & $2.72 \pm 0.07^{\mathrm{c}}$ \\
\hline Sep.01 & 6.60 & 5.20 & 4.50 & 3.60 & 3.40 & 2.70 & 1.50 & 1.30 & 1.50 & $3.37 \pm 0.06^{\mathrm{b}}$ \\
\hline Sep.02 & 7.70 & 4.50 & 3.80 & 3.40 & 3.10 & 2.80 & 1.50 & 1.40 & 1.80 & $3.33 \pm 0.06^{\mathrm{b}}$ \\
\hline Oct.01 & 7.80 & 6.30 & 5.40 & 4.50 & 3.90 & 3.10 & 1.90 & 2.30 & 2.50 & $4.19 \pm 0.05^{\mathrm{a}}$ \\
\hline Oct.02 & 8.40 & 4.90 & 4.40 & 3.90 & 3.60 & 3.40 & 1.90 & 2.00 & 2.40 & $3.88 \pm 0.06^{\mathrm{a}}$ \\
\hline Nov.01 & 4.00 & 2.80 & 2.20 & 2.00 & 2.00 & 1.40 & 0.60 & 0.50 & 0.70 & $1.80 \pm 0.04^{\text {ghi }}$ \\
\hline Nov.02 & 4.10 & 2.70 & 1.90 & 2.00 & 1.80 & 1.20 & 0.70 & 0.40 & 0.60 & $1.71 \pm 0.04^{\text {ghij }}$ \\
\hline Dec.01 & 2.90 & 1.90 & 1.40 & 1.20 & 0.80 & 0.90 & 0.40 & 0.20 & 0.40 & $1.12 \pm 0.05^{\mathrm{k}}$ \\
\hline Dec.02 & 2.80 & 2.10 & 1.30 & 1.10 & 1.00 & 0.80 & 0.40 & 0.20 & 0.40 & $1.12 \pm 0.06^{\mathrm{k}}$ \\
\hline
\end{tabular}

Table 3. Overall mean and SD of different stages of LBF on host plants, Citrus limetta leaves under field conditions

\begin{tabular}{|c|c|c|c|c|c|c|c|c|c|c|}
\hline Months & Eggs & $\begin{array}{c}1^{\text {st }} \\
\text { instar }\end{array}$ & $\begin{array}{c}2^{\text {nd }} \\
\text { instar }\end{array}$ & $\begin{array}{c}3^{\text {rd }} \\
\text { instar }\end{array}$ & $\begin{array}{c}4^{\text {th }} \\
\text { instar }\end{array}$ & $\begin{array}{c}5^{\text {th }} \\
\text { instar }\end{array}$ & upae & $\begin{array}{l}\text { Adult } \\
\text { male }\end{array}$ & $\begin{array}{l}\text { Adult } \\
\text { female }\end{array}$ & Mean \pm SD \\
\hline Jan.01 & 2.80 & 1.80 & 1.30 & 1.00 & 0.80 & 0.90 & 0.30 & 0.10 & 0.30 & $1.03 \pm 0.06^{\mathrm{mn}}$ \\
\hline Jan.02 & 3.10 & 2.10 & 1.40 & 1.20 & 1.00 & 0.80 & 0.40 & 0.30 & 0.50 & $1.20 \pm 0.03^{1 \mathrm{mn}}$ \\
\hline Feb.01 & 3.70 & 2.20 & 1.80 & 1.30 & 1.40 & 1.20 & 0.50 & 0.40 & 0.60 & $1.46 \pm 0.06^{\mathrm{ijkl}}$ \\
\hline Feb.02 & 3.80 & 2.30 & 1.60 & 1.40 & 1.10 & 1.00 & 0.50 & 0.30 & 0.50 & $1.39 \pm 0.05^{\mathrm{jkl}}$ \\
\hline Mar.01 & 5.30 & 3.00 & 2.60 & 2.00 & 1.90 & 1.80 & 0.90 & 0.60 & 0.80 & $2.10 \pm 0.05^{\mathrm{ef}}$ \\
\hline Mar.02 & 4.60 & 3.10 & 2.20 & 2.10 & 1.60 & 1.70 & 0.80 & 0.50 & 0.70 & $1.92 \pm 0.06^{\mathrm{fg}}$ \\
\hline Apr.01 & 5.10 & 3.30 & 2.90 & 2.30 & 2.10 & 2.10 & 1.00 & 0.60 & 0.80 & $2.24 \pm 0.06^{\mathrm{de}}$ \\
\hline Apr.02 & 5.70 & 3.40 & 2.50 & 2.30 & 1.80 & 1.90 & 0.90 & 0.60 & 1.00 & $2.23 \pm 0.04^{\mathrm{de}}$ \\
\hline May.01 & 4.30 & 2.60 & 2.20 & 1.70 & 1.80 & 1.70 & 0.70 & 0.40 & 0.60 & $1.78 \pm 0.05^{\mathrm{gh}}$ \\
\hline May.02 & 4.10 & 2.80 & 2.00 & 1.80 & 1.40 & 1.50 & 0.60 & 0.40 & 0.60 & $1.69 \pm 0.04^{\mathrm{ghi}}$ \\
\hline June.01 & 3.20 & 1.90 & 1.50 & 1.10 & 1.00 & 1.10 & 0.40 & 0.20 & 0.40 & $1.20 \pm 0.06^{\mathrm{Imn}}$ \\
\hline & 3.50 & 2.20 & 1.60 & 1.40 & 1.10 & 1.00 & 0.50 & 0.20 & 0.40 & $05^{\mathrm{jklm}}$ \\
\hline July.01 & 3.40 & 2.00 & 1.60 & 1.20 & 1.10 & 1.00 & 0.40 & 0.30 & 0.50 & $1.28 \pm 0.05^{\mathrm{klm}}$ \\
\hline July.02 & 3.30 & 2.10 & 1.50 & 1.30 & 1.00 & 0.90 & 0.50 & 0.20 & .40 & $1.24 \pm 0.04^{\mathrm{klm}}$ \\
\hline Aug.01 & 5.90 & 3.60 & 3.00 & 2.60 & 2.40 & 2.30 & 1.10 & 0.80 & 1.00 & $2.52 \pm 0.05^{\mathrm{cd}}$ \\
\hline Aug.02 & 5.40 & 3.50 & 2.70 & 2.60 & 1.90 & 2.10 & 1.00 & 0.70 & 0.90 & $2.31 \pm 0.08^{\mathrm{de}}$ \\
\hline Sep.01 & 6.30 & 4.20 & 3.40 & 2.90 & 2.80 & 2.60 & 1.50 & 1.40 & 1.80 & $2.99 \pm 0.06^{\mathrm{b}}$ \\
\hline Sep.02 & 5.80 & 3.90 & 3.20 & 2.90 & 2.20 & 2.30 & 1.40 & 1.10 & 1.50 & $2.70 \pm 0.06^{\mathrm{bc}}$ \\
\hline Oct.01 & 7.40 & 4.80 & 4.10 & 3.80 & 3.40 & 2.80 & 1.70 & 1.60 & 2.00 & $3.51 \pm 0.06^{\mathrm{a}}$ \\
\hline Oct.02 & 6.60 & 4.40 & 3.90 & 3.40 & 2.80 & 2.60 & 1.80 & 1.90 & 2.30 & $3.30 \pm 0.06^{\mathrm{a}}$ \\
\hline Nov.01 & 3.90 & 2.40 & 2.00 & 1.50 & 1.60 & 1.50 & 0.50 & 0.40 & 0.60 & $1.60 \pm 0.03^{\text {hij }}$ \\
\hline Nov.02 & 3.80 & 2.50 & 1.80 & 1.60 & 1.30 & 1.20 & 0.50 & 0.40 & 0.60 & $1.52 \pm 0.05^{\mathrm{hijk}}$ \\
\hline Dec.01 & 2,50 & 1.50 & 1.00 & 0.80 & 0.70 & 0.80 & 0.30 & 0.20 & 0.40 & $0.91 \pm 0.06^{\mathrm{n}}$ \\
\hline Dec.02 & 2.80 & 1.90 & 1.20 & 1.00 & 0.80 & 0.60 & 0.40 & 0.20 & 0.40 & $1.03 \pm 0.02^{\mathrm{mn}}$ \\
\hline
\end{tabular}

Table 4. Overall mean and SD of different stages of LBF on host plants, Citrus reticulata leaves under field conditions

\begin{tabular}{|c|c|c|c|c|c|c|c|c|c|c|}
\hline Months & Eggs & $\begin{array}{c}\mathbf{1}^{\text {st }} \\
\text { instar }\end{array}$ & $\begin{array}{c}\mathbf{2}^{\text {nd }} \\
\text { instar }\end{array}$ & $\begin{array}{c}\mathbf{3}^{\text {rd }} \\
\text { instar }^{\text {instar }}\end{array}$ & $\begin{array}{c}\mathbf{4}^{\text {th }} \\
\text { instar }\end{array}$ & $\begin{array}{c}\mathbf{5}^{\text {th }} \\
\text { instar }\end{array}$ & Pupae & $\begin{array}{c}\text { Adult } \\
\text { male }\end{array}$ & $\begin{array}{c}\text { Adult } \\
\text { female }\end{array}$ & Mean \pm SD \\
\hline Jan.01 & 2.40 & 1.40 & 1.00 & 0.80 & 0.60 & 0.70 & 0.30 & 0.20 & 0.40 & $0.87 \pm 0.03^{\text {mno }}$ \\
\hline Jan.02 & 2.60 & 1.90 & 1.20 & 0.90 & 0.80 & 0.60 & 0.30 & 0.20 & 0.40 & $0.99 \pm 0.03^{\text {Imno }}$ \\
\hline
\end{tabular}




\begin{tabular}{|c|c|c|c|c|c|c|c|c|c|c|}
\hline Feb.01 & 2.80 & 1.80 & 1.50 & 1.30 & 1.10 & 0.90 & 0.50 & 0.30 & 0.50 & $1.19 \pm 0.04^{\mathrm{jkl}}$ \\
\hline Feb.02 & 3.00 & 2.40 & 1.70 & 1.30 & 1.20 & 0.80 & 0.40 & 0.30 & 0.50 & $1.29 \pm 0.03^{\mathrm{ijk}}$ \\
\hline Mar.01 & 4.00 & 2.80 & 2.40 & 1.80 & 1.60 & 1.60 & 0.70 & 0.60 & 0.80 & $1.81 \pm 0.06^{\mathrm{fg}}$ \\
\hline Mar.02 & 4.30 & 3.00 & 2.30 & 1.90 & 1.80 & 1.30 & 0.60 & 0.60 & 0.80 & $1.84 \pm 0.07^{\mathrm{g}}$ \\
\hline Apr.01 & 4.60 & 3.10 & 2.70 & 2.00 & 1.80 & 1.70 & 0.90 & 0.60 & 0.80 & $2.02 \pm 0.06^{\mathrm{ef}}$ \\
\hline Apr.02 & 4.40 & 3.30 & 2.50 & 2.10 & 2.00 & 1.50 & 0.80 & 0.60 & 0.80 & $2.00 \pm 0.05^{\mathrm{ef}}$ \\
\hline May.01 & 3.80 & 2.50 & 2.00 & 1.60 & 1.50 & 1.40 & 0.60 & 0.40 & 0.60 & $1.60 \pm 0.04^{\text {gh }}$ \\
\hline May.02 & 3.30 & 2.80 & 2.10 & 1.70 & 1.60 & 1.10 & 0.50 & 0.40 & 0.60 & $1.57 \pm 0.03^{\text {ghi }}$ \\
\hline June.01 & 2.50 & 1.50 & 1.30 & 1.10 & 0.80 & 0.70 & 0.40 & 0.20 & 0.40 & $0.99 \pm 0.04^{\mathrm{lmno}}$ \\
\hline June.02 & 2.70 & 2.10 & 1.30 & 1.00 & 0.90 & 0.60 & 0.30 & 0.20 & 0.40 & $1.06 \pm 0.06^{\mathrm{klmn}}$ \\
\hline July.01 & 2.60 & 1.60 & 1.40 & 1.20 & 0.90 & 0.80 & 0.40 & 0.30 & 0.50 & $1.08 \pm 0.03^{\mathrm{klmn}}$ \\
\hline July.02 & 2.80 & 2.20 & 1.50 & 1.10 & 1.00 & 0.70 & 0.30 & 0.20 & 0.40 & $1.13 \pm 0.03^{\mathrm{klm}}$ \\
\hline Aug.01 & 4.70 & 3.40 & 3.00 & 2.20 & 1.90 & 1.90 & 0.90 & 0.70 & 0.90 & $2.18 \pm 0.06^{\mathrm{de}}$ \\
\hline Aug.02 & 5.30 & 3.50 & 2.60 & 2.40 & 2.10 & 1.60 & 0.80 & 0.70 & 0.90 & $2.21 \pm 0.05^{\mathrm{de}}$ \\
\hline Sep.01 & 5.60 & 3.90 & 3.30 & 2.50 & 2.10 & 2.20 & 1.40 & 1.10 & 1.30 & $2.60 \pm 0.08^{\mathrm{bc}}$ \\
\hline Sep.02 & 4.90 & 3.70 & 2.90 & 2.60 & 2.40 & 1.90 & 1.30 & 1.10 & 1.30 & $2.46 \pm 0.06^{\mathrm{cd}}$ \\
\hline Oct.01 & 5.30 & 4.40 & 3.80 & 2.80 & 2.40 & 2.30 & 1.50 & 1.50 & 1.70 & $2.86 \pm 0.06^{\mathrm{ab}}$ \\
\hline Oct.02 & 6.20 & 4.00 & 3.50 & 2.90 & 2.70 & 2.30 & 1.60 & 1.50 & 1.90 & $2.96 \pm 0.06^{\mathrm{a}}$ \\
\hline Nov.01 & 3.40 & 2.20 & 1.80 & 1.50 & 1.30 & 1.10 & 0.50 & 0.40 & 0.60 & $1.42 \pm 0.04^{\mathrm{hij}}$ \\
\hline Nov.02 & 3.20 & 2.60 & 1.90 & 1.50 & 1.40 & 1.00 & 0.40 & 0.40 & 0.60 & $1.44 \pm 0.04^{\mathrm{gij}}$ \\
\hline Dec.01 & 2.10 & 1.20 & 0.90 & 0.70 & 0.60 & 0.60 & 0.30 & 0.20 & 0.40 & $0.78 \pm 0.02^{\mathrm{o}}$ \\
\hline Dec.02 & 2.20 & 1.50 & 1.00 & 0.80 & 0.70 & 0.50 & 0.30 & 0.20 & 0.40 & $0.84 \pm 0.04^{\text {no }}$ \\
\hline
\end{tabular}

Table 5. Overall mean and SD of different stages of LBF on host plants, Citrus aurantium leaves under field conditions

\begin{tabular}{|c|c|c|c|c|c|c|c|c|c|c|}
\hline Months & Eggs & $\begin{array}{c}1^{\text {st }} \\
\text { instar }\end{array}$ & $\begin{array}{c}2^{\text {nd }} \\
\text { instar }\end{array}$ & $\begin{array}{c}3^{\text {rd }} \\
\text { instar }\end{array}$ & $\begin{array}{c}4^{\text {th }} \\
\text { instar }\end{array}$ & $\begin{array}{c}5^{\text {th }} \\
\text { instar }\end{array}$ & Pupae & $\begin{array}{c}\text { Adult } \\
\text { male }\end{array}$ & $\begin{array}{l}\text { Adult } \\
\text { female }\end{array}$ & Mean \pm SD \\
\hline Jan.01 & 1.80 & 1.00 & 0.70 & 0.50 & 0.40 & 0.50 & 0.30 & 0.20 & 0.40 & $0.64 \pm 0.01^{\mathrm{pq}}$ \\
\hline Jan.02 & 2.00 & 1.50 & 0.80 & 0.60 & 0.50 & 0.30 & 0.20 & 0.10 & 0.30 & $0.70 \pm 0.21^{\mathrm{opq}}$ \\
\hline Feb.01 & 2.20 & 1.40 & 1.10 & 0.90 & 0.80 & 0.80 & 0.40 & 0.30 & 0.50 & $0.93 \pm 0.03^{1 \mathrm{mno}}$ \\
\hline Feb.02 & 2.50 & 1.80 & 1.10 & 0.90 & 1.00 & 0.50 & 0.40 & 0.30 & 0.50 & $1.00 \pm 0.07^{\mathrm{klmn}}$ \\
\hline Mar.01 & 3.80 & 2.00 & 1.70 & 1.60 & 1.40 & 1.50 & 0.60 & 0.50 & 0.70 & $1.53 \pm 0.05^{\text {fgh }}$ \\
\hline Mar.02 & 2.80 & 2.50 & 1.70 & 1.40 & 1.50 & 1.30 & 0.60 & 0.40 & 0.60 & $1.42 \pm 0.04^{\mathrm{ghi}}$ \\
\hline Apr.01 & 3.90 & 2.20 & 1.90 & 1.80 & 1.60 & 1.70 & 0.70 & 0.50 & 0.70 & $1.67 \pm 0.03^{\text {efg }}$ \\
\hline Apr.02 & 3.00 & 2.70 & 1.90 & 1.60 & 1.60 & 1.50 & 0.70 & 0.60 & 0.80 & $1.60 \pm 0.04^{\text {efgh }}$ \\
\hline May.01 & 2.60 & 1.80 & 1.50 & 1.30 & 1.20 & 1.30 & 0.50 & 0.40 & 0.60 & $1.24 \pm 0.03^{\mathrm{ijk}}$ \\
\hline May.02 & 3.00 & 2.30 & 1.60 & 1.20 & 1.30 & 1.20 & 0.50 & 0.40 & 0.60 & $1.34 \pm 0.05^{\text {hij }}$ \\
\hline June.01 & 1.90 & 1.10 & 0.80 & 0.60 & 0.50 & 0.60 & 0.30 & 0.20 & 0.40 & $0.71 \pm 0.03^{\text {opq }}$ \\
\hline June.02 & 2.20 & 1.60 & 0.90 & 0.80 & 0.70 & 0.40 & 0.30 & 0.30 & 0.40 & $0.83 \pm 0.03^{\text {mnop }}$ \\
\hline July.01 & 2.00 & 1.20 & 0.90 & 0.70 & 0.60 & 0.70 & 0.30 & 0.20 & 0.40 & $0.78 \pm 0.02^{\text {nopq }}$ \\
\hline July.02 & 2.40 & 1.70 & 1.00 & 0.90 & 0.80 & 0.50 & 0.30 & 0.30 & 0.40 & $0.91 \pm 0.03^{\operatorname{lmno}}$ \\
\hline Aug.01 & 3.50 & 2.40 & 2.20 & 2.10 & 1.70 & 1.90 & 0.80 & 0.50 & 0.70 & $1.76 \pm 0.04^{\mathrm{def}}$ \\
\hline Aug.02 & 4.10 & 3.00 & 2.10 & 1.80 & 1.70 & 1.60 & 0.70 & 0.50 & 0.70 & $1.80 \pm 0.05^{\mathrm{de}}$ \\
\hline Sep.01 & 4.40 & 2.80 & 2.40 & 2.30 & 1.80 & 2.10 & 1.20 & 0.90 & 1.10 & $2.11 \pm 0.05^{\mathrm{bc}}$ \\
\hline Sep.02 & 3.70 & 3.50 & 2.40 & 2.10 & 1.90 & 1.60 & 0.90 & 0.90 & 1.10 & $2.01 \pm 0.04^{\mathrm{cd}}$ \\
\hline Oct.01 & 4.80 & 3.30 & 2.70 & 2.60 & 2.10 & 2.40 & 1.40 & 1.20 & 1.60 & $2.46 \pm 0.05^{\mathrm{a}}$ \\
\hline Oct.02 & 3.90 & 3.80 & 2.90 & 2.50 & 2.30 & 2.00 & 1.00 & 1.20 & 1.40 & $2.33 \pm 0.04^{\mathrm{ab}}$ \\
\hline Nov.01 & 2.80 & 1.60 & 1.30 & 1.10 & 1.00 & 1.10 & 0.50 & 0.40 & 0.60 & $1.16 \pm 0.03^{j \mathrm{kl}}$ \\
\hline Nov.02 & 2.40 & 2.10 & 1.40 & 1.10 & 1.10 & 0.60 & 0.40 & 0.30 & 0.50 & $1.10 \pm 0.04^{\mathrm{jklm}}$ \\
\hline Dec.01 & 1.50 & 0.80 & 0.60 & 0.40 & 0.30 & 0.40 & 0.30 & 0.20 & 0.40 & $0.54 \pm 0.02^{\mathrm{q}}$ \\
\hline Dec.02 & 1.80 & 1.30 & 0.70 & 0.50 & 0.40 & 0.40 & 0.20 & 0.10 & 0.30 & $0.63 \pm 0.02^{\mathrm{pq}}$ \\
\hline
\end{tabular}




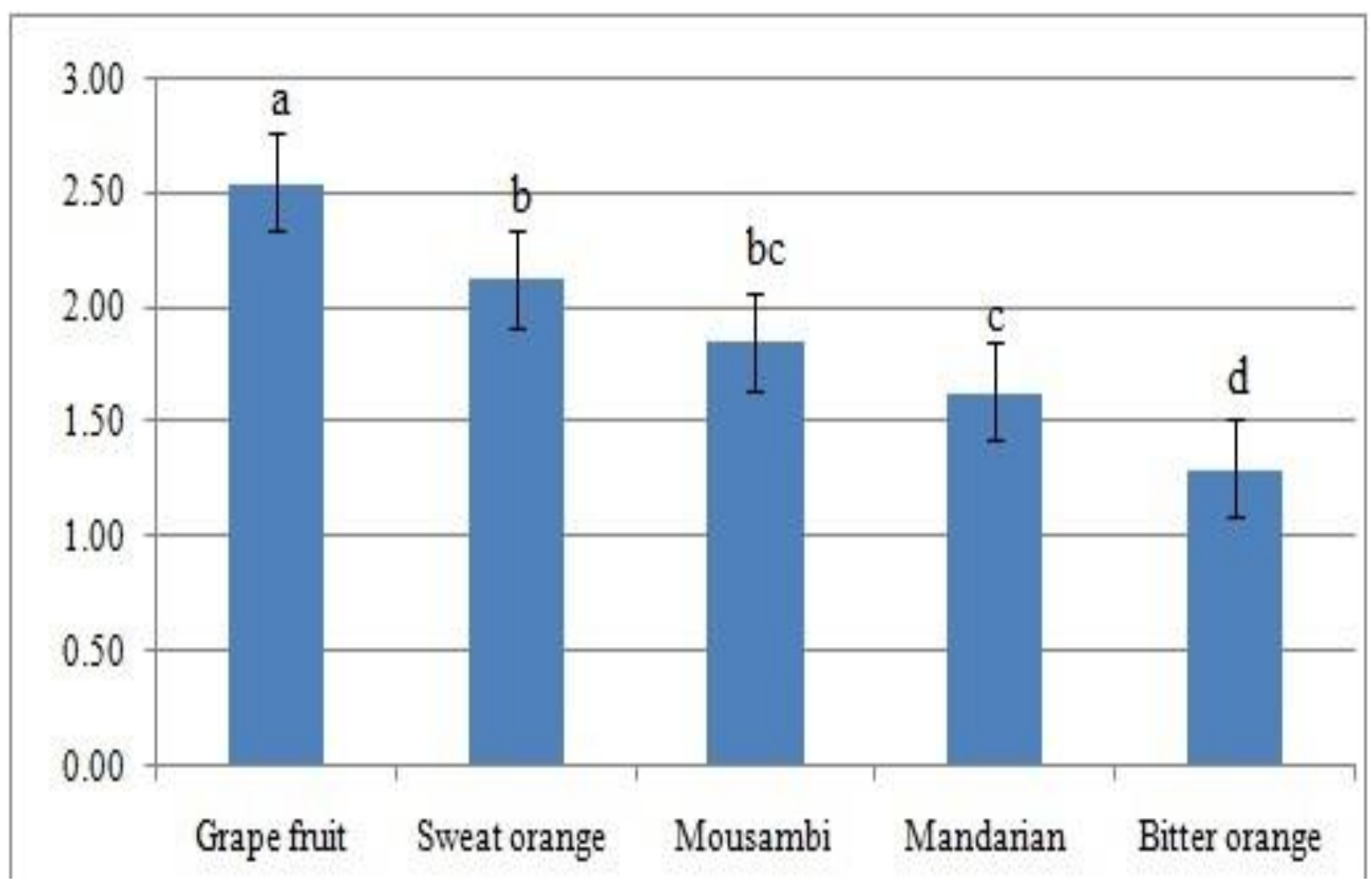

Figure 1. Overall mean of LBF pest population on five different citrus host plants

\section{Discussion}

Citrus fruits are the third largest industry covers about $6 \%$ area compared to other fruits in the world [17]. Citrus orchards are aromatic small shrubs, thorny, and contain evergreen leaves, sufficiently grow at appropriate neither in much humid nor in much tropics [18]. Butterflies play a pivot role in the food chain, as a source of food for spiders, reptiles, predatory insects, birds. In the terrestrial ecosystem they serving as a food source, soil formation, plant-pollinator, and recycling agent. Butterflies are grace creatures and familiar by their marvellous shape, bright colours, diurnal feeding behaviour and attractive flight patterns give pleasure [19]. During host plant survey many insect pests have been found from the five varieties of citrus orchards among them, $P$. demoleus observed destructive agent and major pest of citrus orchards widely use foliage for food, hence sometimes called plague to citrus [20].

Herbivore insect species not only prefer accurate plant species but also feed on several other plant species for their development but LBF insects in old-world range cause the great infestation to lemon orchards and growers are facing economical losses [21]. Our results are with the agreement with the work of [22], he described that the larvae of these insect pests frequently feed on wild as well as a cultivated variety of citrus orchards, especially those plants which come into the category of family Rutaceae from all growing steps of the plants and cause defoliation [23]. Including lemon orchards, these pest insects nourish on different alternate citrus plants at larval stages including, Grapefruits, Sweat oranges, Pomelo, Mousambi, Mandarin oranges, Bitter oranges, Lime, Lemon, Tangerine, etc and harming nursery to grown-up trees, agreement with the work of [10].

LBF population less or greater found throughout the year it is compared with the work of [24], he documented that the acid lime and mandarin plantation are infested by throughout the year but severely during July to August and low temperature and moderate relative humidity favour the activity of the larvae. Several scientific 
approaches show the fluctuation of the population due to climatic and environmental effects of different regions. During present work LBF population was observed at high intensity during the October month this is similar with the approach of [25], they reported a high incidence of pest population at citrus lemon during June to October at India, in same region peak butterfly larval population reported during August to September [26]. While as the initial appearance in March and abruptly breeding period from July to September was reported by [27] and their larvae observed more active up to November then pupal falls in the cold season [28].

In Pakistan, many researchers performed work on different aspects of LBF including; morphology, biology, multiplicity, and distribution of butterflies. [29], they described the activities and effects of butterflies from Karachi and their adjoining areas [30], from Kashmir he documented different behavioural patterns of butterflies and various life aspects of butterflies fauna documented at Chitral by [31]. IPM programs from many years in the parts of the world are involved to provide sufficient measures and to provide proper doorstep facilities to how to combat form insect pest species and minimize the indirect and direct human health hazards without disturbing the ecosystem. Currently, a certain botanical insecticide is used to restrict the growth of destructive insect pests and minimize environmental pollution. Many strategies of IMP are environmentally, biologically, socially, culturally tolerable, and friendly [32].

\section{Conclusion and recommendations}

During field conditioning surveys from different host citrus plants at form lands in the one-year survey study plan, all stages of the LBF pest population were observed form all mentioned verities. It was found that normal temperature is sweat able for their successful growth and development but at high temperatures and coldness decreases their population. In the present study, October month was observed fruitful for their sufficient breeding, hatching, larval growth, and adult mating. Similarly, due to the intensity of coldness less population was observed in December month. Infield condition it was seen that adult male and female LBF can fly at large distances but frequently move back towards their preferred citrus host plants formatting and oviposition purpose and male took initiative for copulations. In present findings, the maximum LBF population was seen on Grapefruit leaves as compared to Sweat orange leaves, Mousambi leaves, Mandarian orange leaves, and Bitter orange leaves. Citrus fruits are a bumper crop of this area but severely harmed by potential insect pest LBF. Presently, in many countries of the world, wide range IMP programs have been launched in response to provide general awareness for growers to recognize and identify harmful pests and their natural enemies in their fruit-bearing valuable crops but in this regard, it is strictly needed for dynamic management over $P$. demoleus. Previously there is no scientific documentary of such type of work performed in this area, this kind of research work on five alternate host plants was performed the first time and it will be an informative tool for citrus growers for this region.

\section{Authors' contributions}

The experiments conceived and designated: HA Sahito \& Bhugro Mal, Experiments performed: WM Mangrio \& T Kousar, Data analysed: WM Mangrio, Contributed analyses, tools, materials: $\mathrm{ZH}$ Shah \& FA Jatoi: Wrote the paper: WM Mangrio \& HA Sahito.

\section{Acknowledgment}

The authors gratefully acknowledge the citrus fruit farm landowners for providing cheerfully support during the field survey. Again authors are highly pleased to their loyal research guide Prof. Dr. Hakim Ali Sahito for their restless efforts, and his 
expertise gave us like third eye pathways in a present scientific investigation.

\section{References}

1. Singh D (1969). Citrus industry in India today. Indian J Hort 13(2): 1632.

2. Hayes WB (1957). Fruit growing in India. Kitabistan Pbl. Ltd Allahbad India.

3. Shah MA (2004). Citrus cultivation in NWFP. Proce the 1st Inter Conf on Citri Uni o Agri Faisalabad 3-39.

4. Tiple AD, Khurad AM \& Dennis RLH (2011). Butterfly larval host plant used in a tropical urban context. Life history associations, herbivore and landscape factors. J Insect Sci 11: 121.

5. Liu W, Wang $\mathrm{Y} \& \mathrm{Xu} \mathrm{R}$ (2006). Habitat utilization by ovipositing females and larvae of the Marsh fritillary (Euphydryas aurinia) in a mosaic of meadows and croplands. $J$ Insect Con 10: 351-360.

6. Thomas JA, Simox DJ, Wardlaw JC, Elms WG, Hochberg ME \& Clark RT (1998). Effects of latitude, altitude, and climate on the habitat and conservation of the endangered butterfly Maculinea arion and its Myrmica and host. J Sect Conserv 2(3): 9-46.

7. Borges RM, Gowda V \& Zacharias M (2003). Butterfly pollination and high contrast visual signals in a low-density distylous plant. Oecologica 136: 571573.

8. Homoziak T, Nicholas T \& Homziak J (2006). P. demoleus (Lepidoptera: Papilionidae). A new record for the United States, Commonwealth of Puerto Rico. Florida Entomol 89: 485-488.

9. Shivankar VJ \& Singh S (1999). Insect pests of citrus integrated approach for their management. Inten Agri 11: 17-22.

10. Butani DK \& Jotwani MG (1975). Trends in the control of insect pests of fruit crops in India. Pesticides 9(4):
139-149. Hill S \& Dennis (1981). Agricultural insect pests of the tropics and their control. Skegness lincs England, pp 746.

11. Mangrio WM, Sahito HA, Chandio NH, Kousar T, Shah ZH, Khaskheli NA \& Jatoi FA (2019). Food and feed consumption of lemon butterfly, $P$. demoleus under laboratory conditions. Pure and Appli Bio 9(1): 340-351.

12. Pathak KN \& Rizvi PQ (2002). Agespecific life table of $P$. demoleus on different hosts. Anna Plant Prot Sci 10: 375-376.

13. Narayanamma VL \& Savithri $P$ (2002). Seasonal abundance of the citrus butterfly, $P$. demoleus Linn. On sathgudy sweet orange and Tenali acid lime. J of App Zoo Rese 13(1): 54 56.

14. Yunus M \& Munir M (1972). Host plants and host preference of lemon butterfly, $P$. demoleus Linn. Caterpillars. Pak J Zool 4: 231-232.

15. Malik JM (1970). Notes on the butterflies of Pakistan in the collection of Zoological survey department Karachi Part I. Rec. Zoo Sur of Paki 2(2): 25-54.

16. Kunte K (2006). Additions to known larval host plants of Indian butterflies. $J$ Bombay Nat Hist Soc 103(1): 119-120.

17. Sarada G, Gopal K, Venkata RKT, Lakshmi LM \& Nagalakshmi T (2014). Citrus butterfly (P. demoleus Linnaeus), biology and management. A Review. J Agric All Sci 3(1): 17-25.

18. Hill S \& Dennis (1981). Agricultural insect pests of the tropics and their control. Skegness,lincs, England, pp 746

19. Javed I (1978). Preliminary report of butterflies of district Rawalpindi and Islamabad. Biologia 2: 238-247.

20. Chatterjee H, Ghosh J \& Senapati SK (2000). Influence of important weather parameters on population fluctuation on major insect pest of mandarin orange (Citrus reticulata 
Blanco) at Darjeeling district of West Bengal, India. J Ento Res 24(3): 229233.

21. Pakistan agricultural research council (2003). Integrated pest management. Updated March 19, 2003, Retrieved June 9, 2006 from http://www. parc.gov.pk/ipm.html.

22. Atwal AS (1964). Insect pests of citrus in Punjab. Biology and control of citrus caterpillar. $P$ demoleus $\mathrm{L}$. (Lepidoptera: Papilionidae). Punjab Horti J 4: 40-44.

23. Narayanamma VL, Savithri P \& Rao AR (2001). Influence of citrus butterfly $P$. demoleus $\mathrm{L}$. damage on growth parameters of the Sweet orange host plant. Indian J Plant Prot 29: 140-141.

24. Beattie GAC (2004). Citrus leaf miner. NSW Agriculture and Fisheries. Agfactt H2 AE 41-48.

25. Shravan MHV, Karuppaiah V, Sharma SK \& Singh RS (2010). Population dynamics of lemon butterfly $(P$. demoleus L.) in Bael (Aegle marmelos) as influenced by abiotic factors in arid regions of Rajasthan. Indian J Arid Hort 5(1-2): 50-52.

26. Alturi JB, Ramana SPV \& Reddi CS (2002). Life history of $P$. demoleus
(Lepidoptera: Rhopalocera: Papilionidae) from India. $J$ Natl Taiwan Mus 55: 27-32.

27. Mastumoto K (1996). Establishment of $P$. demoleus L. (Papilionidae) in Java. J Lepid Soc 50: 139-140.

28. Nandini D, Raghuwanshi A \& Shrivastava VK (2012). Life cycle, population index and feeding activities of the lime butterfly, $P$. demoleus (Lepidoptera: Rhopalocera: Papilionidae). Trends Biosci 5(1): 3134.

29. Swinhow C (1987). On the Lepidoptera of Karachi and its neighborhood. J Bombay Nat Hist Soc 269-280.

30. Doherty W (1986). List of butterflies taken in Kashmir. $J$ Asiatic Soc Bangladesh 55: 103-140.

31. Leslic \& Evans WH (1933). The butterflies of Balochistan. J Bombay Nat Hist Soc 36(1): 196-209.

32. Kenmore PE, Litsinger JA, Bandong JP, Santiago AC \& Salac MM (1985). Philippine rice farmers and insecticides in thirty years of growing dependency and new options for change, pp 98-108. 